\title{
Almost Automorphic Functions of Order $n$ and Applications to Dynamic Equations on Time Scales
}

\author{
Gisèle Mophou, ${ }^{1,2}$ Gaston Mandata N'Guérékata, ${ }^{3}$ and Aril Milce ${ }^{1,4}$ \\ ${ }^{1}$ Laboratoire CEREGMIA, Université des Antilles et de la Guyane, Campus Fouillole, 97159 Pointe-à-Pitre, Guadeloupe \\ ${ }^{2}$ Laboratoire MAINEGE, Université Ouaga 3S, 06 BP 10347, Ouagadougou 06, Burkina Faso \\ ${ }^{3}$ Department of Mathematics, Morgan State University, Baltimore, MD 21251, USA \\ ${ }^{4}$ Département de Mathématiques, École Normale Supérieure, Université d'État d'Haïti, Rue Monseigneur Guilloux, \\ Port-au-Prince, Haiti \\ Correspondence should be addressed to Gisèle Mophou; gisele.mophou@univ-ag.fr
}

Received 30 August 2014; Revised 24 November 2014; Accepted 4 December 2014; Published 31 December 2014

Academic Editor: Qi-Ru Wang

Copyright (c) 2014 Gisèle Mophou et al. This is an open access article distributed under the Creative Commons Attribution License, which permits unrestricted use, distribution, and reproduction in any medium, provided the original work is properly cited.

We revisit the notion on almost automorphic functions on time scales given by Lizama and Mesquita (2013). Then we present the notion of almost automorphic functions of order $n$. Finally, we apply this notion to study the existence and uniqueness and the global stability of almost automorphic solution of first order to a dynamical equation with finite time varying delay.

\section{Introduction}

The concept of time scales was initiated in 1988 by Hilger in his outstanding Ph.D. thesis [1]. The purpose of such theory was to unify both continuous and discrete analysis. Consequently, using time scales in studying dynamic systems prevents from proving results separately for differential equations and difference equations. Since then, several papers were devoted to dynamical systems on time scales [2-8]. We refer also readers to the excellent book by Bohner and Peterson [9] and their edited book [10] which contains high quality contributions to the theory.

It was natural to study almost periodic time scales as well as almost periodic differential equations on almost periodic time scales [3]. Our initial motivation for the current study comes from [4] where the authors studied the existence and exponential stability of almost periodic solutions of a neutraltype BAM neural network with delays on time scales, using exponential dichotomy of linear dynamical systems.

Recently Lizama and Mesquita introduced the notion of almost automorphic functions on time scales in their work [11]. The purpose of this paper is twofold. First we would like to revisit Lizama and Mesquita's paper in light of the following remarks.
Let $\mathbb{T}$ be a time scale. It is said to be invariant under translations if

$$
\Pi=\{\tau \in \mathbb{R}: t \mp \tau \in \mathbb{T}, \forall t \in \mathbb{T}\} \neq\{0\} .
$$

We prove in Lemma 25 that $\Pi \subset \mathbb{T} \Leftrightarrow 0 \in \mathbb{T}$.

However, we observe that the inclusion may be strict. Indeed, let us consider the time scale $P_{a, b}=\bigcup_{k=-\infty}^{\infty}[k(a+$ $b), k(a+b)+a$ ], where $0<a<b$; it is obviously invariant under translations, and it contains 0 and $a$ but not $-a$. Then $a \notin \Pi$. This also proves that the invariant under translations time scales $P_{a, b}$ is not symmetric.

For this reason, several results in [11] hold only if the time scale is symmetric.

Secondly we would like to study the existence and stability of almost automorphic solutions of the following linear dynamic system with finite delay:

$$
\begin{gathered}
x_{i}^{\Delta}(t)=-a_{i}(t) x_{i}(t)+\sum_{j=1}^{m} b_{i j}(t) f_{j}\left(x_{j}\left(t-\tau_{i j}(t)\right)\right)+I_{i}(t), \\
t \in \mathbb{T}, i=1,2, \ldots, m, \\
x_{i}(s)=\varphi(s), \quad s \in[-\theta, 0]_{\mathbb{T}}, i=1,2, \ldots, m,
\end{gathered}
$$


where $\mathbb{T}$ is an appropriate time scale, $m \geq 2$ is the number of neurons in the network, $x_{i}(t)$ denote the activation of the $i$ th neuron at time $t$, and $a_{i}$ represents the rate with which the $i$ th neurons will rest their potential to the resting state in isolation when they are disconnected from the network and the external inputs at time $t$. The $m \times m$ matrix $B(t)=\left(b_{i j}(t)\right)$ represents the connection strengths between neurons at time $t ; b_{i j}$ is an element of feedback templates at time $t ; f_{j}$ is the activation function. $\tau_{i j}$ is the transmission delay at time $t$ and satisfies $t-\tau_{i j} \in \mathbb{T} ; I_{i}(t)$ denote the bias of the $i$ th neuron at time $t, \theta=\max _{(i, j)} \sup _{t \in \mathbb{\mathbb { T }}} \tau_{i j}(t)$ and $[a, b]_{\mathbb{T}}=\{t \mid t \in[a, b] \cap \mathbb{T}\}$.

We organized our paper as follows. In Section 2, we recall some definitions and recent results on time scales. In Section 3, we present properties of almost automorphic functions on symmetric time scales along with a composition theorem. In Section 4, we introduce and present elementary properties of almost automorphic functions on time scales of order $n$ and study differentiation and integration of such functions in Section 5 and superposition of operators on the space of such functions in Section 6. Finally in Section 7, we study the existence, uniqueness, and global stability of system (2).

\section{Preliminaries}

In this section we recall some definitions and recent results on time scales.

Definition 1. A time scale is an arbitrary nonempty closed subset of real numbers.

Definition 2. Let $\mathbb{T}$ be a time scale. The forward and backward jump operators $\sigma, \rho: \mathbb{T} \rightarrow \mathbb{T}$ and the graininess $\mu: \mathbb{T} \rightarrow$ $[0,+\infty)$ are defined, respectively, by

$$
\begin{gathered}
\sigma(t)=\inf \{s \in \mathbb{T}: s>t\}, \quad \rho(t)=\sup \{s \in \mathbb{T}: s<t\} \\
\mu(t)=\sigma(t)-t .
\end{gathered}
$$

In Definition 5, we put inf $\emptyset=\sup \mathbb{T}$ and $\sup \emptyset=\inf \mathbb{T}$.

Definition 3. Let $\mathbb{T}$ be time scales.

(i) A point $t \in \mathbb{T}$ is called right-dense if $t<\sup \mathbb{T}$ and $\sigma(t)=t$.

(ii) A point $t \in \mathbb{T}$ is called left-dense if $t>\inf \mathbb{T}$ and $\rho(t)=t$.

Definition 4 (see [9]). A function $f: \mathbb{T} \rightarrow \mathbb{R}$ is called regulated provided its right-sided limits exist (finite) at all rightdense points in $\mathbb{T}$ and its left-sided limits exist (finite) at all left-dense points in $\mathbb{T}$.

Definition 5 (see [9]). A function $f: \mathbb{T} \rightarrow \mathbb{R}$ is called rdcontinuous provided it is continuous at right-dense points in $\mathbb{T}$ and its left-sided limits exist (finite) at left-dense points in $\mathbb{T}$.
We will denote the set of rd-continuous function $f: \mathbb{T} \rightarrow$ $\mathbb{R}$ by $C_{\text {rd }}=C_{\text {rd }}(\mathbb{T}, \mathbb{R})$. From now on, we define the set $\mathbb{T}^{k}$ by

$$
\mathbb{T}^{k}= \begin{cases}\mathbb{T} \backslash[\rho(\sup \mathbb{T}), \sup \mathbb{\mathbb { T }}] & \text { if } \sup \mathbb{\mathbb { N }}<\infty \\ \mathbb{T} & \text { if } \sup \mathbb{\mathbb { V }}=\infty .\end{cases}
$$

Definition 6 (see [9]). Let $f: \mathbb{T} \rightarrow \mathbb{R}$ be a function and $t \in \mathbb{T}^{k}$. We define $f^{\Delta}(t)$ to be the number (provided it exists) with the property that, given any $\epsilon>0$, there exists a neighborhood $U$ of $t$ such that

$$
\begin{array}{r}
\left|f(\sigma(t))-f(s)-f^{\Delta}(t)[\sigma(t)-s]\right| \leq \epsilon|\sigma(t)-s| \\
\forall s \in U .
\end{array}
$$

We call $f^{\Delta}(t)$ the delta (or Hilger) derivative of $f$ at $t$.

Moreover, we say that $f$ is delta (or Hilger) differentiable (or in short differentiable) on $\mathbb{T}^{k}$ provided $f^{\Delta}(t)$ exists for all $t \in \mathbb{T}^{k}$. The function $f^{\Delta}: \mathbb{T}^{k} \rightarrow \mathbb{R}$ is called the (delta) derivative of $f$ on $\mathbb{T}^{k}$.

Next we recall some easy and useful relationships concerning the delta derivative.

Theorem 7 (see [9]). Assume $f: \mathbb{T} \rightarrow \mathbb{R}$ is a function and let $t \in \mathbb{T}^{k}$. Then one has the following.

(i) If $f$ is differentiable at $t$, then $f$ is continuous at $t$.

(ii) If $f$ is continuous at $t$ and $t$ is right-scattered, then $f$ is differentiable at $t$ with

$$
f^{\Delta}(t)=\frac{f(\sigma(t))-f(t)}{\mu(t)} .
$$

(iii) If $t$ is right-dense, then $f$ is differentiable at $t$ if and only if the limit

$$
\lim _{s \rightarrow t} \frac{f(t)-f(s)}{t-s}
$$

exists as a finite number. In this case

$$
f^{\Delta}(t)=\lim _{s \rightarrow t} \frac{f(t)-f(s)}{t-s} .
$$

(iv) If $f$ is differentiable at $t$, then

$$
f(\sigma(t))=f(t)+\mu(t) f^{\Delta}(t) .
$$

Theorem 8 (see [9]). Assume $f, g: \mathbb{T} \rightarrow \mathbb{R}$ are differentiable at $t \in \mathbb{T}^{k}$. Then

(i) the sum $f+g: \mathbb{T} \rightarrow \mathbb{R}$ is differentiable at $t$ with

$$
(f+g)^{\Delta}(t)=f^{\Delta}(t)+g^{\Delta}(t) ;
$$

(ii) for any constant $\alpha, \alpha f: \mathbb{T} \rightarrow \mathbb{R}$ is differentiable at $t$ with

$$
(\alpha f)^{\Delta}(t)=\alpha f^{\Delta}(t)
$$


(iii) the product $f g: \mathbb{T} \rightarrow \mathbb{R}$ is differentiable at $t$ with

$$
\begin{aligned}
(f g)^{\Delta}(t) & =f^{\Delta}(t) g(t)+f(\sigma(t)) g^{\Delta}(t) \\
& =f(t) g^{\Delta}(t)+f^{\Delta}(t) g(\sigma(t)) .
\end{aligned}
$$

We define higher order derivatives of a function on time scale in the usual way.

Definition 9 (see [9]). For a function $f: \mathbb{T} \rightarrow \mathbb{R}$ one will talk about the second derivative $f^{\Delta \Delta}$ provided $f^{\Delta}$ is differentiable on $\mathbb{T}^{k^{2}}:=\left(\mathbb{T}^{k}\right)^{k}$ with derivative $f^{\Delta \Delta}:=\left(f^{\Delta}\right)^{\Delta}: \mathbb{T}^{k^{2}} \rightarrow \mathbb{R}$. Similarly one defines higher order derivatives $f^{\Delta^{n}}: \mathbb{T}^{k^{n}} \rightarrow$ $\mathbb{R}$. Finally, for $t \in \mathbb{T}$, one denotes $\sigma^{2}(t):=\sigma(\sigma(t))$ and $\rho^{2}(t):=\rho(\rho(t))$, and $\sigma^{n}(t)$ and $\rho^{n}(t)$ are defined accordingly. For convenience one also puts $\rho^{0}(t)=\sigma^{0}(t)=t$ and $\mathbb{T}^{k^{0}}=\mathbb{T}$.

Theorem 10 (see [9] (Leibniz formula)). Let $S_{k}^{(n)}$ be the set consisting of all possible strings of length $n$, containing exactly $k$ times $\sigma$ and $n-k$ times $\Delta$. If $f^{\Lambda}$ exists for all $\Lambda \in S_{k}^{(n)}$, then

$$
(f g)^{\Delta^{n}}=\sum_{k=0}^{n}\left(\sum_{\Lambda \in S_{k}^{(n)}} f^{\Lambda}\right) g^{\Delta^{k}}
$$

holds for all $n \in \mathbb{N}$.

The following results on chain rule can be found in [9].

Theorem 11 (chain rule). Let $f: \mathbb{R} \rightarrow \mathbb{R}$ be continuously differentiable and suppose $g: \mathbb{T} \rightarrow \mathbb{R}$ is delta differentiable on $\mathbb{T}^{k}$. Then $f \circ g: \mathbb{T} \rightarrow \mathbb{R}$ is delta differentiable and the formula

$$
(f \circ g)^{\Delta}(t)=\left[\int_{0}^{1} f^{\prime}\left(g(t)+h \mu(t) g^{\Delta}(t)\right) d h\right] \times g^{\Delta}(t)
$$

holds for all $t \in \mathbb{T}^{k}$.

Theorem 12 (chain rule). Assume that $v: \mathbb{T} \rightarrow \mathbb{R}$ is strictly increasing and $\widetilde{\mathbb{T}}=\nu(\mathbb{T})$ is a time scale. Let $\omega: \widetilde{\mathbb{T}} \rightarrow \mathbb{X}$, where $\mathbb{X}$ is a Banach space. If $\nu^{\Delta}(t)$ and $\omega^{\widetilde{\Delta}}(t)$ exist for $t \in \mathbb{T}^{k}$, then

$$
(\omega \circ v)^{\Delta}=v^{\Delta} \cdot \omega^{\tilde{\Delta}} \circ v,
$$

where ${ }^{\tilde{\Delta}}$ denote the ${ }^{\Delta}$ in $\widetilde{\mathbb{T}}^{k}$.

Definition 13. If $a \in \mathbb{T}$, sup $\mathbb{T}=+\infty$, and $f$ is a $C_{\text {rd }}$ function on $[a,+\infty)$, then the improper integral of $f$ is defined by

$$
\int_{a}^{\infty} f(t) \Delta t=\lim _{b \rightarrow+\infty} \int_{a}^{b} f(t) \Delta t
$$

provided this limit exists. In this case, the improper integral is said to converge.

Lemma 14 (see [4]). Let $a \in \mathbb{T}^{k}, b \in \mathbb{T}$, and assume that $f: \mathbb{T} \times \mathbb{T}^{k} \rightarrow \mathbb{R}$ is continuous at $(t, t)$, where $t \in \mathbb{T}^{k}$ with $t>a$. Assume also that $f^{\Delta}(t, \cdot)$ is rd-continuous on $[a, \sigma(t)]$. Suppose that, for each $\epsilon>0$, there exists a neighborhood $U$ of $\tau \in[a, \sigma(t)]$ such that

$$
\left|f(\sigma(t), \tau)-f(s, \tau)-f^{\Delta}(t, \tau)[\sigma(t)-s]\right| \leq \epsilon|\sigma(t)-s|
$$

$\forall s \in U$,

where $f^{\Delta}$ denotes the derivative of $f$ with respect to the first variable. Then

$$
\begin{gathered}
g(t)=\int_{a}^{t} f(t, \tau) \Delta \tau \quad \text { implies } \\
g^{\Delta}(t)=\int_{a}^{t} f^{\Delta}(t, \tau) \Delta \tau+f(\sigma(t), t) .
\end{gathered}
$$

We now present some definitions and results useful for the study of some dynamical systems.

Definition 15 (see [9]). One says that a function $p: \mathbb{T} \rightarrow \mathbb{R}$ is regressive provided

$$
1+\mu(t) p(t) \neq 0, \quad \forall t \in \mathbb{T}^{k} .
$$

The set of all regressive functions will be denoted by $\mathscr{R}=$ $\mathscr{R}(\mathbb{T}, \mathbb{R})$.

Definition 16 . One defines the set $\mathscr{R}^{+}$of all positively regressive elements of $\mathscr{R}$ by

$$
\begin{aligned}
\mathscr{R}^{+} & =\mathscr{R}^{+}(\mathbb{T}, \mathbb{R}) \\
& =\{p \in \mathscr{R}: 1+\mu(t) p(t)>0 \forall t \in \mathbb{T}\} .
\end{aligned}
$$

Definition 17 (see [9]). If $p \in \mathscr{R}$, then one defines the generalized exponential function by

$$
e_{p}(t, s)=\exp \left(\int_{s}^{t} \xi_{\mu(\tau)}(p(\tau)) \Delta \tau\right) \quad \text { for } t, s \in \mathbb{T} \text {, }
$$

where the cylinder transformation $\xi_{h}: \mathbb{C}_{h} \rightarrow \mathbb{Z}_{h}$ is given by

$$
\xi_{h}((z))=\frac{1}{h} \log (1+z h)
$$

where $\log$ is the principal logarithm function. For $h=0$, we define $\xi_{0}(z)=z$ for all $z \in \mathbb{C}$.

The generalized exponential functions have the following properties.

Lemma 18 (see [9]). Assume that $p, q: \mathbb{T} \rightarrow \mathbb{R}$ are two regressive functions. Then

(i) $e_{0}(t, s) \equiv 1$ and $e_{p}(t, t) \equiv 1$;

(ii) $e_{p}(t, s)=1 / e_{p}(s, t)=e_{\ominus p}(s, t)$;

(iii) $e_{p}(t, s) e_{p}(s, r)=e_{p}(t, r)$;

(iv) $\left[e_{p}(t, s)\right]^{\Delta}=p(t) e_{p}(t, s)$. 
Lemma 19 (see [9]). Assume that $p \in \mathscr{R}^{+}$. Then

(i) $e_{p}(t, s)>0$, for all $t, s \in \mathbb{T}$;

(ii) if $p(t) \leq q(t)$ for all $t \geq s, t, s \in \mathbb{T}$, then $e_{p}(t, s) \leq$ $e_{q}(t, s)$ for all $t \geq s$.

Lemma 20 (see [9]). If $p \in \mathscr{R}$ and $a, b, c \in \mathbb{T}$, then

(i) $\left[e_{p}(c, \cdot)\right]^{\Delta}=-p\left[e_{p}(c, \cdot)\right]^{\sigma}$,

(ii) $\int_{a}^{b} p(t) e_{p}(c, \sigma(t)) \Delta t=e_{p}(c, a)-e_{p}(c, b)$ for all $t \geq s$.

Proposition 21 (see [9]). Let $p: \mathbb{T} \rightarrow \mathbb{R}$ be rd-continuous and regressive, $t_{0} \in \mathbb{T}$, and $y_{0} \in \mathbb{R}$. Then the unique solution of the initial value problem

$$
y^{\Delta}(t)=p(t) y(t)+h(t), \quad y\left(t_{0}\right)=y_{0}
$$

is given by

$$
y(t)=e_{p}\left(t, t_{0}\right)+\int_{t_{0}}^{t} e_{p}(t, \sigma(s)) h(s) \Delta s .
$$

We now present some definitions about matrix-valued functions on $\mathbb{T}$.

Definition 22. Let $A$ be an $m \times m$ matrix-valued function on $\mathbb{T}$. One says that $A$ is $\mathrm{rd}$-continuous on $\mathbb{T}$ if each entry of $A$ is rd-continuous on $\mathbb{T}$. One denotes by $\mathscr{C}_{\text {rd }}\left(\mathbb{T}, \mathbb{R}^{m \times m}\right)$ the class of all rd-continuous $m \times m$ matrix-valued functions on $\mathbb{T}$.

We say that $A$ is delta differentiable on $\mathbb{T}$ if each entry of $A$ is delta differentiable on $\mathbb{T}$. And in this case, we have

$$
A(\sigma(t))=A(t)+\mu(t) A^{\Delta}(t) .
$$

Definition 23. An $m \times m$ matrix-valued function $A$ is called regressive if

$$
I+\mu(t) A(t) \quad \text { is invertible } \forall t \in \mathbb{T}^{k} .
$$

The class of all such regressive rd-continuous functions is denoted by

$$
\mathscr{R}\left(\mathbb{T}, \mathbb{R}^{m \times m}\right) .
$$

\section{Almost Automorphic Functions of Order $n$ on Time Scales}

From now on, $(\mathbb{X},\|\cdot\|)$ is a (real or complex) Banach space.

Definition 24 (see [11]). A time scale $\mathbb{T}$ is called invariant under translations if

$$
\Pi=\{\tau \in \mathbb{R}: t \mp \tau \in \mathbb{T}, \forall t \in \mathbb{T}\} \neq\{0\} .
$$

Lemma 25. Let $\mathbb{T}$ be an invariant under translations time scale. Then one has

(i) $\Pi \subset \mathbb{T} \Leftrightarrow 0 \in \mathbb{T}$;

(ii) $\Pi \cap \mathbb{T}=\emptyset \Leftrightarrow 0 \notin \mathbb{T}$.
Proof

(i) In view of the definition of $\Pi$, if $0 \in \mathbb{T}$, then for all $\tau \in$ $\Pi$ we have $\tau \in \mathbb{T}$. Thus, $\Pi \subset \mathbb{T}$. Conversely, assume that $\Pi \subset \mathbb{T}$. Then, for any $\tau \in \Pi$, we have $0=\tau-\tau \in \mathbb{T}$. Thus $0 \in \mathbb{T}$.

(ii) It is clear that if $\Pi \cap \mathbb{T}=\emptyset$ then $0 \notin \mathbb{T}$. Now assume that $0 \notin \mathbb{T}$. If $\tau \in \Pi \cap \mathbb{T}$, then we have $t-\tau \in \mathbb{T}$ for any $t \in \mathbb{T}$; particularly, for $t=\tau$, we have $\tau-\tau=0 \in \mathbb{T}$. This contradicts the fact that $0 \notin \mathbb{\mathbb { T }}$. Thus $\Pi \cap \mathbb{\mathbb { V }}=\emptyset$.

We have the following properties of the points in $\mathbb{T}$, forward jump operator, and the graininess function when the time scales are invariant under translations.

Lemma 26 (see [2]). Let $\mathbb{T}$ be an invariant under translation time scale. If $t$ is right-dense (resp., right-scattered), then for every $h \in \Pi, t+h$ is right-dense (resp., right-scattered).

Lemma 27 (see [2]). Let $\mathbb{T}$ be an invariant under translations time scale and $h \in \Pi$. Then

(i) $\sigma(t+h)=\sigma(t)+h$ and $\sigma(t-h)=\sigma(t)-h$, for every $t \in \mathbb{T}$;

(ii) $\mu(t+h)=\mu(t)=\mu(t-h)$, for every $t \in \mathbb{T}$.

Remark 28. As we pointed out in Section 1 time scales invariant under translations are not automatically symmetric. Since almost automorphic functions are defined on symmetric domains, some definitions and results in [11] on these functions will be given with additional assumption on the time scale. More precisely we will assume that the time scale is symmetric and invariant under translations.

Definition 29. Let $\mathbb{X}$ be Banach space and let $\mathbb{T}$ be a symmetric time scale which is invariant under translations. Then the rd-continuous function $f: \mathbb{T} \rightarrow \mathbb{X}$ is called almost automorphic on $\mathbb{T}$ if for every sequence $\left(s_{n}\right)$ on $\Pi$, there exists a subsequence $\left(\tau_{n}\right) \subset\left(s_{n}\right)$ such that

$$
\bar{f}(t)=\lim _{n \rightarrow \infty} f\left(t+\tau_{n}\right)
$$

is well defined for each $t \in \mathbb{T}$ and

$$
\lim _{n \rightarrow \infty} \bar{f}\left(t-\tau_{n}\right)=f(t)
$$

for each $t \in \mathbb{T}$.

We denote by $A A_{\mathbb{T}}(\mathbb{X})$ the space of all almost automorphic functions on time scales $f: \mathbb{T} \rightarrow \mathbb{X}$.

Remark 30. In view of Lemma 27 , if $\mathbb{T}$ is a symmetric time scale which is invariant under translations then the graininess function $\mu: \mathbb{T} \rightarrow \mathbb{R}_{+}$is an almost automorphic function.

We have the following properties.

Theorem 31. Let $\mathbb{T}$ be a symmetric time scale which is invariant under translation. Assume that the rd-continuous functions 
$f, g: \mathbb{T} \rightarrow \mathbb{X}$ are almost automorphic on $\mathbb{\mathbb { T }}$. Then the following assertions hold:

(i) $f+g$ is almost automorphic on time scales;

(ii) $\lambda f$ is almost automorphic on $\mathbb{\mathbb { V }}$ for every scalar $\lambda$;

(iii) for each $l \in \Pi$, the function $f_{l}: \mathbb{T} \rightarrow \mathbb{X}$ defined by $f_{l}(t)=f(t+l)$ is almost automorphic on time scales;

(iv) $\widehat{f}: \mathbb{T} \rightarrow \mathbb{X}$ defined by $\widehat{f}(t)=f(-t)$ is almost automorphic on time scales;

(v) $\sup _{t \in \mathbb{T}}\|f(t)\|<\infty$; that is, $f$ is a bounded function;

(vi) $\sup _{t \in \mathbb{T}}\|\bar{f}(t)\|=\sup _{t \in \mathbb{T}}\|f(t)\|$, where

$\bar{f}(t)=\lim _{n \rightarrow \infty} f\left(t+\tau_{n}\right), \quad \lim _{n \rightarrow \infty} \bar{f}\left(t-\tau_{n}\right)=f(t)$.

Proof. See [11]. [11].

We have the following remark on the property given in

Remark 32. Notice that

(i) in order to give a sense to (iii), we consider $l$ as an element of $\Pi$ instead of $l \in \mathbb{T}$ as in [11];

(ii) we need the symmetry of the time scale $\mathbb{T}$ to obtain that $-t \in \mathbb{T}$ if $t \in \mathbb{T}$, that is, to give a sense to the definition of $\widehat{f}$ in (iv).

Remark 33. The space $A A_{\mathbb{T}}(\mathbb{X})$ equipped with the norm $\sup _{t \in \mathbb{T}}\|f(t)\|$ is a Banach space (see [11] pp. 2280).

Lemma 34. If $f \in A A_{\mathbb{T}}(\mathbb{X})$, the range $R_{f}=\{f(t): t \in \mathbb{T}\}$ is relatively compact in $\mathbb{X}$.

Proof. Let $a \in \mathbb{T}$ be fixed and let $\left(x_{n}^{\prime}\right)_{n}$ be a sequence in $R_{f}$. Then, for any $n \in \mathbb{N}$, there is $t_{n}^{\prime} \in \mathbb{T}$ such that $x_{n}^{\prime}=f\left(t_{n}^{\prime}\right)$. By invariance under translations of $\mathbb{T}$, for each $n \in \mathbb{N}$, we can find $\alpha_{n}^{\prime} \in \Pi$ such that $t_{n}^{\prime}=a+\alpha_{n}^{\prime}$. Hence, for all $n \in \mathbb{N}$, we have $x_{n}^{\prime}=f\left(a+\alpha_{n}^{\prime}\right)$. Since $f$ is almost automorphic on time scale, there exists a subsequence $\left(\alpha_{n}\right)_{n}$ of $\left(\alpha_{n}^{\prime}\right)_{n}$ such that

$$
\lim _{n \rightarrow \infty} f\left(a+\alpha_{n}\right)=\bar{f}(a) \text {. }
$$

Thus, the subsequence $\left(x_{n}=f\left(a+\alpha_{n}\right)\right)$ converges to $\bar{f}(a)$. Therefore, $R_{f}$ is relatively compact in $\mathbb{X}$.

Theorem 35 (see [11]). Let $\mathbb{T}$ be a symmetric time scale which is invariant under translations. Let also $u: \mathbb{T} \rightarrow \mathbb{C}$ and $f$ : $\mathbb{T} \rightarrow \mathbb{X}$ be two almost automorphic functions on time scales. Then the function $u f: \mathbb{T} \rightarrow \mathbb{X}$ defined by $(u f)(t)=u(t) f(t)$ is almost automorphic on time scales.

Theorem 36 (see [11]). Let $\mathbb{T}$ be a symmetric time scale which is invariant under translations and let $\left(f_{n}\right)$ be a sequence of almost automorphic functions such that $\lim _{n \rightarrow+\infty} f_{n}(t)=f(t)$ converges uniformly for each $t \in \mathbb{T}$. Then, $f$ is an almost automorphic function.
Theorem 37 (see [11]). Let $\mathbb{T}$ be a symmetric time scale which is invariant under translations and let $\mathbb{X}$ and $\mathbb{Y}$ be Banach spaces. Suppose $f: \mathbb{T} \rightarrow \mathbb{X}$ is an almost automorphic function on time scales and $\phi: \mathbb{X} \rightarrow \mathbb{Y}$ is a continuous function; then the composite function $\phi \circ f: \mathbb{T} \rightarrow \mathbb{Y}$ is almost automorphic on time scales.

Definition 38 (see [11]). Let $\mathbb{X}$ be a (real or complex) Banach space and let $\mathbb{T}$ be a symmetric time scale which is invariant under translations. Then a rd-continuous function $f: \mathbb{T} \times$ $\mathbb{X} \rightarrow \mathbb{X}$ is called almost automorphic on $t \in \mathbb{T}$ for each $x \in \mathbb{X}$ if for every sequence $\left(s_{n}\right)$ on $\Pi$, there exists a subsequence $\left(\tau_{n}\right) \subset\left(s_{n}\right)$ such that

$$
\bar{f}(t, x)=\lim _{n \rightarrow \infty} f\left(t+\tau_{n}, x\right)
$$

is well defined for each $t \in \mathbb{T}, x \in \mathbb{X}$ and

$$
\lim _{n \rightarrow \infty} \bar{f}\left(t-\tau_{n}, x\right)=f(t, x)
$$

for each $t \in \mathbb{T}$ and $x \in \mathbb{X}$.

Theorem 39 (see [11]). Let $\mathbb{~ b e ~ a ~ s y m m e t r i c ~ t i m e ~ s c a l e ~ w h i c h ~}$ is invariant under translations and let $f, g: \mathbb{\mathbb { X }} \times \mathbb{X} \rightarrow \mathbb{X}$ be almost automorphic functions on time scale in $t$ for each $x$ in $\mathbb{X}$. Then the following assertions hold:

(i) $f+g$ is almost automorphic on time scale in $t$ for each $x$ in $\mathbb{X}$;

(ii) $\lambda f$ is almost automorphic function on time scale in $t$ for each $x$ in $\mathbb{X}$, where $\lambda$ is an arbitrary scalar;

(iii) $\sup _{t \in \mathbb{T}}\|f(t, x)\|=M_{x}<\infty$, for each $x \in \mathbb{X}$;

(iv) $\sup _{t \in \mathbb{T}}\|\bar{f}(t, x)\|=N_{x}<\infty$, for each $x \in \mathbb{X}$, where $\bar{f}$ is the function in Definition 38.

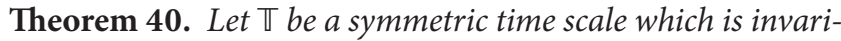
ant under translations and let $f: \mathbb{I} \times \mathbb{X} \rightarrow \mathbb{X}$ be almost automorphic functions on time scale in $t$ for each $x$ in $\mathbb{X}$ and satisfy Lipschitz condition in $x$ uniformly in $t$; that is,

$$
\|f(t, x)-f(t, y)\| \leq L\|x-y\|,
$$

for all $x, y \in \mathbb{X}$. Assume that $\phi: \mathbb{T} \rightarrow \mathbb{X}$ almost automorphic on time scale. Then the function $U: \mathbb{T} \rightarrow \mathbb{X}$ defined by $U(t)=$ $f(t, \phi(t))$ is almost automorphic on time scale.

We can now introduce the notion of almost automorphic functions of order $n$ on time scales.

\section{Almost Automorphic Functions of Order $n$ on Time Scales}

We denote by $C_{\text {rd }}^{n}(\mathbb{T}, \mathbb{X})$ the linear space of all functions $f: \mathbb{T} \rightarrow \mathbb{X}$ that are $n$th differentiable on $\mathbb{T}$ and $f^{\Delta^{n}}$ is rd-continuous. We denote by $B_{\mathrm{rd}}^{n}(\mathbb{T}, \mathbb{X})$ the subspace of $C_{\text {rd }}^{n}(\mathbb{T}, \mathbb{X})$ consisting of such function $f: \mathbb{T} \rightarrow \mathbb{X}$ for which

$$
\sup _{t \in \mathbb{T}}\left(\sum_{i=0}^{n}\left\|f^{\Delta^{i}}(t)\right\|\right)<+\infty,
$$


where $f^{\Delta^{i}}$ denotes the $i$ th derivative of $f, i=1,2, \ldots, n, f^{\Delta^{0}}=$ $f$, and $f^{\Delta^{1}}=f^{\Delta}$. In the space $B_{\mathrm{rd}}^{n}(\mathbb{T}, \mathbb{X})$ we introduce the norm

$$
\|f\|_{n}=\sup _{t \in \mathbb{T}}\left(\sum_{i=0}^{n}\left\|f^{\Delta^{i}}(t)\right\|\right), \quad \text { for } f \in B_{\mathrm{rd}}^{n}(\mathbb{T}, \mathbb{X}) .
$$

Then we have the following.

Proposition 41. $B_{\mathrm{rd}}^{n}(\mathbb{T}, \mathbb{X})$ equipped with the norm defined above is a Banach space.

Proof. It's clear that $B_{\mathrm{rd}}^{n}(\mathbb{T}, \mathbb{X})$ is a linear space and that (37) is a norm on $B_{\text {rd }}^{n}(\mathbb{X})$. Now, let $\left(f_{j}\right)_{j}$ be a Cauchy sequence in $B_{\mathrm{rd}}^{n}(\mathbb{\mathbb { T }}, \mathbb{X})$. Then for any $\epsilon>0$, there exists $N \in \mathbb{N}$ such that, for all $j \in \mathbb{N}, j>N$, we have

$$
\begin{array}{r}
\left\|f_{j+p}-f_{j}\right\|_{n}=\sup _{t \in \mathbb{T}}\left(\sum_{i=0}^{n}\left\|f_{p+j}^{\Delta^{i}}(t)-f_{j}^{\Delta^{i}}(t)\right\|\right) \leq \epsilon \\
\forall p \in \mathbb{N} .
\end{array}
$$

In other words, given $\epsilon>0$, there is $N \in \mathbb{N}$ such that, for all $j \in \mathbb{N}, j>N$,

$$
\sup _{p \in \mathbb{N}}\left\|f_{j+p}-f_{j}\right\|_{n}=\sup _{\substack{p \in \mathbb{N} \\ t \in \mathbb{T}}}\left(\sum_{i=0}^{n}\left\|f_{p+j}^{\Delta^{i}}(t)-f_{j}^{\Delta^{i}}(t)\right\|\right) \leq \epsilon .
$$

In particular, for all $t \in \mathbb{T},\left(f_{j}^{\Delta^{i}}(t)\right), i=0,1, \ldots, n$, are Cauchy sequences in $\mathbb{X}$ which is a Banach space. Thus if we denote by $f$ the limit of $f_{j}^{\Delta^{0}}(t)$, we have that

$$
f_{j}^{\Delta^{i}}(t) \longrightarrow f^{\Delta^{i}}(t), \quad i=0,1, \ldots, n .
$$

Since for $i=0,1, \ldots, n$,

$$
\left\|f_{p+j}^{\Delta^{i}}(t)-f_{j}^{\Delta^{i}}(t)\right\| \leq \epsilon, \quad \forall j, p \in \mathbb{N}, \forall t \in \mathbb{T},
$$

passing to the limit in these above $n+1$ relations as $p \rightarrow+\infty$, we obtain for $i=0,1, \ldots, n$,

$$
\left\|f_{j}^{\Delta^{i}}(t)-f^{\Delta^{i}}(t)\right\| \leq \epsilon, \quad \forall j \in \mathbb{N}, \forall t \in \mathbb{T} .
$$

This means that, for all $j \in \mathbb{N}$,

$$
\left\|f_{j}-f\right\|_{n} \leq \sup _{t \in \mathbb{\mathbb { T }}}\left(\sum_{i=0}^{n}\left\|f_{j}^{\Delta^{i}}(t)-f^{\Delta^{i}}(t)\right\|\right) \leq \epsilon
$$

which on the one hand proves that $f \in B_{\mathrm{rd}}^{n}(\mathbb{T}, \mathbb{X})$ since for any $j \in \mathbb{N}$,

$$
\sum_{i=0}^{n}\left\|f^{\Delta^{i}}(t)\right\| \leq\left\|f_{j}-f\right\|_{n}+\left\|f_{j}\right\|_{n}, \quad \forall t \in \mathbb{T},
$$

and on the other hand shows that

$$
f_{j} \longrightarrow f \quad \text { as } j \longrightarrow+\infty .
$$

Definition 42. Let $\mathbb{X}$ be a (real or complex) Banach space and let $\mathbb{T}$ be a symmetric time scale which is invariant under translations. Then a rd-continuous function $f: \mathbb{T} \times \mathbb{X} \rightarrow \mathbb{X}$ is called almost automorphic if $f(t, x)$ is almost automorphic in $t \in \mathbb{Z}$ uniformly for each $x \in B$, where $B$ is any bounded subset of $\mathbb{X}$.

We denote by $A A(\mathbb{\mathbb { X }} \times \mathbb{X}, \mathbb{X})$ the space of all almost automorphic functions on time scales $f: \mathbb{T} \times \mathbb{X} \rightarrow \mathbb{X}$.

Definition 43. A function $f \in C_{\mathrm{rd}}^{n}(\mathbb{T}, \mathbb{X})$ is said to be $C_{\mathrm{rd}^{-}}^{n}$ almost automorphic (briefly $C_{\mathrm{rd}}^{n}$-a.a.), if $f, f^{\Delta^{i}}$ belong to $A A_{\mathbb{W}}(\mathbb{X})$ for all $i=1, \ldots, n$.

Denote by $A A_{\mathbb{T}}^{(n)}(\mathbb{X})$ the set of $\mathscr{C}^{n}$-a.a. functions.

Directly from the above definitions it follows that $A A_{\mathbb{T}}^{(n+1)}(\mathbb{X}) \subset A A_{\mathbb{T}}^{(n)}(\mathbb{X})$. Moreover, putting $n=0$, we have $A A_{\mathbb{T}}^{(0)}(\mathbb{X})=A A_{\mathbb{T}}(\mathbb{X})$.

Lemma 44. We have $A A_{\mathbb{T}}^{(n)}(\mathbb{X}) \subset B_{\mathrm{rd}}^{n}(\mathbb{T}, \mathbb{X})$.

Proof. It is straightforward from the definition of an almost automorphic function on time scales (see Theorem 31).

Proposition 45. A linear combination of $C_{\mathrm{rd}}^{n}$-a.a. functions is $a C_{\mathrm{rd}}^{n}$-a.a. function. Moreover, let $\mathbb{X}$ be a Banach space over the field $\mathbb{K}(\mathbb{K}=\mathbb{R}$ or $\mathbb{C})$. Let $f, g \in A A_{\mathbb{T}}^{(n)}(\mathbb{X}), v \in A A_{\mathbb{T}}^{(n)}(\mathbb{K})$, and $\lambda \in \mathbb{K}$. Assume that $\nu^{\Lambda}$ exists for all $\Lambda \in S_{k}^{(n)}$ and is almost automorphic on time scale. Then the following functions are also in $A A_{\mathbb{T}}^{(n)}(\mathbb{X})$ :

(i) $f+g$,

(ii) $\lambda f$,

(iii) $v f$,

(iv) $f_{a}(t):=f(t+a)$, where $a \in \Pi$ is fixed.

Proof. For the proof of (i) and (ii), one proceeds as in [11]. To prove (iii), we use the Leibnitz formula on time scales, the definition, and the properties of an almost automorphic function; we get the result easily.

Now, let us prove (iv). For any $a \in \Pi$, if we consider the function $v: \mathbb{T} \rightarrow \mathbb{R}$ defined by $v(t)=a+t$, then we have $f_{a}(t)=(f \circ v)(t)$, for all $t$ in $\mathbb{T}$. It is clear that $v$ is strictly increasing, $v(\mathbb{T})=\mathbb{T}$, and $v^{\Delta}(t)=1$, for all $t \in \mathbb{T}$. Using Theorem 12, we obtain $\left(f_{a}\right)^{\Delta}(t)=v^{\Delta}(t) \cdot\left(f^{\Delta} \circ v\right)(t)=$ $f^{\Delta}(v(t))=f^{\Delta}(a+t)=f_{a}^{\Delta}(t)$ for each $t \in \mathbb{T}$. Hence, for $f^{\Delta}$ being an almost automorphic function on time scale, we deduce that $\left(f_{a}\right)^{\Delta}=f_{a}^{\Delta}$ is almost also automorphic on time scale. Similarly, we prove that $\left(f_{a}\right)^{\Delta^{i}}$ is almost automorphic for $i=$ $1,2, \ldots, n$. Thus, $f_{a} \in A A_{\mathbb{T}}^{n}(\mathbb{X})$.

Theorem 46. If a sequence $\left(f_{p}\right)_{p \in \mathbb{N}}$ of $C_{\mathrm{rd}}^{n}-a . a$. functions is such that $\left\|f_{p}-f\right\|_{n} \rightarrow 0$ as $k \rightarrow+\infty$, then $f$ is $C_{\mathrm{rd}}^{n}-a . a$. function. 
Proof. From the assumption, it is clear that $f \in B_{\mathrm{rd}}^{n}(\mathbb{T}, \mathbb{X})$. Moreover, $f_{p}^{\Delta^{i}} \rightarrow f^{\Delta^{i}}$ uniformly on $\mathbb{T}$ for each $i=0, \ldots, n$. Thus Theorem 36 allows us to say that $f$ is a $C_{\mathrm{rd}}^{n}$-a.a. function.

Corollary 47. $A A_{\mathbb{T}}^{(n)}(\mathbb{X})$ considered with norm (37) turns out to be a Banach space.

Proof. In view of Proposition 45 and Lemma 44, $A A_{\mathbb{T}}^{(n)}(\mathbb{X})$ is a linear subspace of $B_{\mathrm{rd}}^{n}(\mathbb{T}, \mathbb{X})$. Let $\left(f_{p}\right), f_{p} \in A A_{\mathbb{T}}^{(n)}(\mathbb{X}), p \in \mathbb{N}$, be a Cauchy sequence. Then there exists $f \in B_{\mathrm{rd}}^{n}(\mathbb{T}, \mathbb{X})$ such that $\lim _{k \rightarrow \infty}\left\|f_{p}-f\right\|_{n}=0$. By Theorem 46, $f \in A A_{\mathbb{T}}^{(n)}(\mathbb{X})$, so it is a Banach space.

\section{Differentiation and Integration}

The first result in this section gives a sufficient condition which guarantees that the derivative of a function $f \in$ $A A_{\mathbb{T}}^{(n)}(\mathbb{X})$ is also a $C_{\mathrm{rd}}^{n}$-a.a. function.

Theorem 48. Let $\mathbb{T}$ be a symmetric time scale which is invariant under translations. Let also $\mathbb{X}$ be a Banach space and $f: \mathbb{T} \rightarrow \mathbb{X}$ an almost automorphic function on $\mathbb{T}$. Assume that $f$ is $\Delta$-differentiable on $\mathbb{T}$ and $f^{\Delta}$ is uniformly continuous. Then $f^{\Delta}$ is also almost automorphic on time scales.

Proof. Assume that the points of $\mathbb{T}$ are right-dense. Then for $\mathbb{T}$ being invariant under translations, we obtain $\mathbb{T}=\mathbb{R}$. Hence $f^{\Delta}=f^{\prime}$ and since $f^{\Delta}$ is uniformly continuous, it follows from Theorem 2.4.1 in [12] that $f^{\Delta}$ is almost automorphic on time scale.

Now, let us suppose that $\mathbb{T}$ has at least a right-scattered point $t_{0}$; then we have

$$
f^{\Delta}\left(t_{0}\right)=\frac{f\left(\sigma\left(t_{0}\right)\right)-f\left(t_{0}\right)}{\mu\left(t_{0}\right)} .
$$

Given a sequence $\left(\alpha_{n}^{\prime}\right) \in \Pi$, since $f$ is almost automorphic, there is a subsequence $\left(\alpha_{n}\right)_{n}$ such that

$$
\begin{aligned}
\lim _{n \rightarrow \infty} f^{\Delta}\left(t_{0}+\alpha_{n}\right) & =\lim _{n \rightarrow \infty} \frac{f\left(\sigma\left(t_{0}+\alpha_{n}\right)\right)-f\left(t_{0}+\alpha_{n}\right)}{\mu\left(t_{0}+\alpha_{n}\right)} \\
& =\lim _{n \rightarrow \infty} \frac{f\left(\sigma\left(t_{0}\right)+\alpha_{n}\right)-f\left(t_{0}+\alpha_{n}\right)}{\mu\left(t_{0}\right)} \\
& =\frac{\bar{f}\left(\sigma\left(t_{0}\right)\right)-\bar{f}\left(t_{0}\right)}{\mu\left(t_{0}\right)} \\
& =g\left(t_{0}\right)
\end{aligned}
$$

since Lemma 27 holds. On the other hand, we have

$$
\begin{aligned}
\lim _{n \rightarrow \infty} g\left(t_{0}-\alpha_{n}\right) & =\lim _{n \rightarrow \infty} \frac{\bar{f}\left(\sigma\left(t_{0}+\alpha_{n}\right)\right)-\bar{f}\left(t_{0}+\alpha_{n}\right)}{\mu\left(t_{0}+\alpha_{n}\right)} \\
& =\lim _{n \rightarrow \infty} \frac{\bar{f}\left(\sigma\left(t_{0}\right)+\alpha_{n}\right)-\bar{f}\left(t_{0}+\alpha_{n}\right)}{\mu\left(t_{0}\right)}
\end{aligned}
$$

$$
\begin{aligned}
& =\frac{f\left(\sigma\left(t_{0}\right)\right)-f\left(t_{0}\right)}{\mu\left(t_{0}\right)} \\
& =f^{\Delta}\left(t_{0}\right) .
\end{aligned}
$$

The proof is completed.

Theorem 49. If $f \in A A_{\mathbb{T}}^{(n)}(\mathbb{X})$ and $f^{\Delta^{n+1}}$ is uniformly continuous, then $f^{\Delta} \in A A_{\mathbb{T}}^{(n)}(\mathbb{X})$.

Proof. In view of Theorem 48, we have $f^{\Delta^{n+1}} \in A A_{\mathbb{T}}(\mathbb{X})$. This means that $f$ is in $A A_{\mathbb{T}}^{(n+1)}(\mathbb{X})$. Then it follows that $f^{\Delta} \in$ $A A_{\mathbb{T}}^{(n)}(\mathbb{X})$.

Similarly as in [12], we introduce some useful notations in order to facilitate the proof.

Notation 1. Let $\mathbb{~ b e ~ a ~ s y m m e t r i c ~ t i m e ~ s c a l e ~ w h i c h ~ i s ~ i n v a r i a n t ~}$ under translations. If $f: \mathbb{T} \rightarrow \mathbb{X}$ is a function and a sequence $\alpha=\left(\alpha_{n}\right) \subset \Pi$ is such that

$$
\lim _{n \rightarrow \infty} f\left(t+\alpha_{n}\right)=g(t) \quad \text { pointwise on } \mathbb{T},
$$

we will write $T_{s} f=g$.

Remark 50 (see [12]). Consider the following.

(i) $T_{s}$ is a linear operator.

Given a fixed sequence $\alpha=\left(\alpha_{n}\right) \subset \Pi$, the domain of $T_{s}$ is $D\left(T_{s}\right)=\left\{f: \mathbb{T} \rightarrow \mathbb{X}\right.$ such that $T_{s} f$ exists $\}$. $D\left(T_{s}\right)$ is a linear set.

(ii) Let us write $-s=\left(-\alpha_{n}\right)$ and suppose that $f \in D\left(T_{s}\right)$ and $T_{s} f \in D\left(T_{-s}\right)$. The product operator $A_{s}=T_{-s} T_{s} f$ is well defined. It is also a linear operator.

(iii) $A_{s}$ maps bounded functions into bounded functions, and for an almost authorphic function on time scale $f$, we get $A_{s} f=f$.

Now we are ready to enunciate and prove Bohl-Bohr's type theorem known from the literature for almost automorphic functions on time scale. The proof is inspired by the proof of Theorem 2.4.4 in [12].

Theorem 51. Let $\mathbb{T}$ be a symmetric time scale containing zero and invariant under translations. Let also $f \in A A_{\mathbb{T}}(\mathbb{X})$. One considers the function $F: \mathbb{T} \rightarrow \mathbb{X}$ defined by $F(t)=\int_{0}^{t} f(s) \Delta s$. Then $F \in A A_{\mathbb{T}}(\mathbb{X})$ if and only if $R_{F}$ is relatively compact in $\mathbb{X}$.

Proof. In view of Lemma 34, it suffices to prove that $F \in$ $A A_{\mathbb{T}}(\mathbb{X})$ if $R_{F}$ is relatively compact in $\mathbb{X}$.

Assume that $R_{F}$ is relatively compact in $\mathbb{X}$ and let $\left(s_{n}^{\prime \prime}\right) \subset$ $\Pi$. Then there exists a subsequence $\left(s_{n}^{\prime}\right)$ of $\left(s_{n}^{\prime \prime}\right)$ such that

$$
\begin{aligned}
& \lim _{n \rightarrow \infty} f\left(t+s_{n}^{\prime}\right)=g(t), \\
& \lim _{n \rightarrow \infty} g\left(t-s_{n}^{\prime}\right)=f(t),
\end{aligned}
$$


pointwise on $\mathbb{T}$, and

$$
\lim _{n \rightarrow \infty} F\left(s_{n}^{\prime}\right)=\alpha_{1}
$$

for some $\alpha_{1} \in \mathbb{X}$.

We get, for every $t \in \mathbb{T}$,

$$
\begin{aligned}
F\left(t+s_{n}^{\prime}\right) & =\int_{0}^{t+s_{n}^{\prime}} f(r) \Delta r \\
& =\int_{0}^{s_{n}^{\prime}} f(r) \Delta r+\int_{s_{n}^{\prime}}^{t+s_{n}^{\prime}} f(r) \Delta r \\
& =F\left(s_{n}^{\prime}\right)+\int_{s_{n}^{\prime}}^{t+s_{n}^{\prime}} f(r) \Delta r .
\end{aligned}
$$

Making the change of variable $\delta=r-s_{n}^{\prime}$, we obtain

$$
F\left(t+s_{n}^{\prime}\right)=F\left(s_{n}^{\prime}\right)+\int_{0}^{t} f\left(\delta+s_{n}^{\prime}\right) \Delta \delta
$$

If we apply the Lebesgue dominated theorem to this latter identity, we get

$$
\lim _{n \rightarrow \infty} F\left(t+s_{n}^{\prime}\right)=\alpha_{1}+\int_{0}^{t} g(\sigma) \Delta \sigma,
$$

for each $t \in \mathbb{T}$. Let us observe that the range of the function $G(t)=\alpha_{1}+\int_{0}^{t} g(r) \Delta r$ is also relatively compact and

$$
\sup _{t \in \mathbb{T}}\|(G(t))\| \leq \sup _{t \in \mathbb{T}}\|F(t)\|
$$

so that we can extract a subsequence $\left(s_{n}\right)$ of $\left(s_{n}^{\prime}\right)$ such that

$$
\lim _{n \rightarrow \infty} G\left(-s_{n}\right)=\alpha_{2}
$$

for some $\alpha_{2} \in \mathbb{X}$. Now we can write

$$
G\left(t-s_{n}\right)=G\left(-s_{n}\right)+\int_{0}^{t} g\left(r-s_{n}\right) \Delta r
$$

so that

$$
\lim _{n \rightarrow \infty} G\left(t-s_{n}\right)=\alpha_{2}+\int_{0}^{t} f(r) \Delta r=\alpha_{2}+F(t) .
$$

Let us prove now that $\alpha_{2}=0$. Using Notation 1 we get

$$
A_{s} F=\alpha_{2}+F
$$

where $s=\left(s_{n}\right)$. Now it is easy to observe that $F$ and $\alpha_{2}$ belong to the domain of $A_{s}$; therefore $A_{s} F$ is also in the domain of $A_{s}$ and we deduce the equation

$$
A_{s}^{2} F=A_{s} \alpha_{2}+A_{s} F=\alpha_{2}+\alpha_{2}+F=2 \alpha_{2}+F \text {. }
$$

We can continue indefinitely the process to get

$$
A_{s}^{n} F=n \alpha_{2}+F, \quad \forall n=1,2, \ldots
$$

But we have

$$
\sup _{t \in \mathbb{T}}\left\|A_{s}^{n} F(t)\right\| \leq \sup _{t \in \mathbb{T}}\|F(t)\|
$$

and $F(t)$ is a bounded function.

This leads to contradiction if $\alpha_{2} \neq 0$. Hence, $\alpha_{2}=0$, and using Remark 50, we have $A_{s} F=F$, so $F$ is almost automorphic. The proof is complete.

Theorem 52. If $f \in A A_{\mathbb{T}}(\mathbb{X})$ and the range $R_{F}$ is relatively compact, then $F \in A A_{\mathbb{T}}^{(1)}(\mathbb{X})$.

Proof. If $f \in A A_{\mathbb{T}}(\mathbb{X})$ and the range $R_{F}$ is relatively compact, then in view of Theorem 51, $F \in A A_{\mathbb{T}}(\mathbb{X})$. Since $F(t)=$ $\int_{0}^{t} f(s) \Delta s, F^{\Delta}(t)=f(t)$, for $t \in \mathbb{T}$. Thus $F^{\Delta} \in A A_{\mathbb{T}}(\mathbb{X})$ and, consequently, $F \in A A_{\mathbb{T}}^{(1)}(\mathbb{X})$.

Theorem 52 is a special case of the following.

Theorem 53. If $f \in A A_{\mathbb{T}}^{(n)}(\mathbb{X})$ and the range $R_{F}$ is relatively compact then $F \in A A_{\mathbb{T}}^{(n+1)}(\mathbb{X})$.

Proof. If $f \in A A_{\mathbb{T}}^{(n)}(\mathbb{X})$ and the range $R_{F}$ is relatively compact then in view of Theorem 51, $F \in A A_{\mathbb{T}}(\mathbb{X})$. Therefore, $F^{\Delta}=$ $f \in A A_{\mathbb{T}}^{(n)}(\mathbb{X})$. This means that $F \in A A_{\mathbb{T}}^{(n+1)}(\mathbb{X})$.

Corollary 54. If $f^{\Delta} \in A A_{\mathbb{T}}^{(n)}(\mathbb{X})$ and the range $R_{f}$ is relatively compact, then $f \in A A_{\mathbb{T}}^{(n+1)}(\mathbb{X})$.

Proof. We know that $f(t)=f(0)+\int_{0}^{t} f^{\Delta}(s) \Delta s$, for each $t \in \mathbb{T}$. In view of Theorem 53, we have $f \in A A_{\mathbb{T}}^{(n+1)}(\mathbb{X})$.

\section{Superposition Operators}

In this section, $\mathbb{X}_{1}$ and $\mathbb{X}_{2}$ are two Banach spaces.

Proposition 55. Let $A: \mathbb{X}_{1} \rightarrow \mathbb{X}_{2}$ be a bounded linear operator and $f \in A A_{\mathbb{T}}^{(n)}\left(\mathbb{X}_{1}\right)$. Then we have Af $\in A A_{\mathbb{T}}^{(n)}\left(\mathbb{X}_{2}\right)$.

Proof. Since $A$ is a bounded linear operator, we have $(A f)^{\Delta^{n}}=$ $A f^{\Delta^{n}}$. Therefore, observing the fact that $f^{\Delta^{i}} \in A A_{\mathbb{T}}\left(\mathbb{X}_{1}\right)$ for each $i=0,1, \ldots, n$ because $f \in A A_{\mathbb{T}}^{(n)}\left(\mathbb{X}_{1}\right)$, it follows from Theorem 37 that $A f^{\Delta^{i}} \in A A_{\mathbb{T}}\left(\mathbb{X}_{2}\right)$, for all $i=0,1, \ldots, n$. Hence $A f \in A A_{\mathbb{T}}^{(n)}\left(\mathbb{X}_{2}\right)$.

For every $f \in A A_{\mathbb{T}}^{(n)}\left(\mathbb{X}_{1}\right)$, we define the function $G: \mathbb{T} \rightarrow$ $\mathbb{X}_{2}$ as follows:

$$
G(t)=\int_{0}^{t} A f(s) \Delta s
$$

where $A: \mathbb{X}_{1} \rightarrow \mathbb{X}_{2}$ is a bounded linear operator. We have the following.

Corollary 56. Let $A: \mathbb{X}_{1} \rightarrow \mathbb{X}_{2}$ be a bounded linear operator with a relatively compact range. Then $\mathbb{X}_{2}$-valued function $G$ defined above is in $A A_{\mathbb{T}}^{(n+1)}\left(\mathbb{X}_{2}\right)$. 
Proof. According to Proposition 55, Af $\in A A_{\mathbb{T}}^{(n)}\left(\mathbb{X}_{2}\right)$ for every $f \in A A_{\mathbb{T}}^{(n)}\left(\mathbb{X}_{1}\right)$. Since $A$ is a bounded linear operator, the range $R_{A}$ of the operator $A$ contains the range $R_{G}$ of $G$. Hence $R_{G}$ is relatively compact and it follows from Theorem 53 that $G \in A A_{\mathbb{T}}^{(n+1)}\left(\mathbb{X}_{2}\right)$.

Remark 57. In Corollary 56, if operator $A$ is compact (or of the finite rank), the stated result holds.

Now we will consider the superposition of operator (the autonomous case) acting on the space $A A_{\mathbb{T}}^{(n)}(\mathbb{X})$. Using this fact we will prove the following result with the Fréchet derivative.

Theorem 58. If $\phi \in \mathscr{C}^{1}(\mathbb{R}, \mathbb{R})$ and $f \in A A_{\mathbb{T}}^{(1)}(\mathbb{R})$, then $\phi \circ f \in$ $A A_{\mathbb{T}}^{(1)}(\mathbb{R})$.

Proof. First, we observe that the result holds if for $n=0$, in view of Theorem 37, we have that $\phi \circ f \in A A_{\mathbb{T}}(\mathbb{R})$ if $\phi \in$ $\mathscr{C}^{1}(\mathbb{R}, \mathbb{R})$ and $f \in A A_{\mathbb{T}}(\mathbb{R})$. So, it suffices to show that $(\phi$ 。 $f)^{\Delta} \in A A_{\mathbb{T}}(\mathbb{R})$ to complete the proof of the theorem.

By Theorem 11, for each $t \in \mathbb{T}$, we have

$$
(\phi \circ f)^{\Delta}(t)=\left[\int_{0}^{1} \phi^{\prime}\left(f(t)+h \mu(t) f^{\Delta}(t)\right) d h\right] f^{\Delta}(t) .
$$

Since $f \in A A_{\mathbb{T}}^{(1)}(\mathbb{R})$ and $\mu \in A A_{\mathbb{T}}\left(\mathbb{R}_{+}\right)$we have that, for any $h \in[0,1]$, the function $t \mapsto f(t)+h \mu(t) f^{\Delta}(t)$ belongs to $A A_{\mathbb{T}}(\mathbb{R})$. Therefore for $\phi^{\prime}$ being continuous, Theorem 37 allows us to say that the function $t \mapsto \phi^{\prime}\left(f(t)+h \mu(t) f^{\Delta}(t)\right)$ is also in $A A_{\mathbb{J}}(\mathbb{R})$ for any $h \in[0,1]$ and consequently the function $t \mapsto \int_{0}^{1} \phi^{\prime}\left(f(t)+h \mu(t) f^{\Delta}(t)\right) d h$ is $A A_{\mathbb{W}}(\mathbb{R})$. It then follows from Theorem 35 that $(\phi \circ f)^{\Delta} \in A A_{\mathbb{T}}(\mathbb{R})$ since $f^{\Delta} \in A A_{\mathbb{T}}(\mathbb{R})$.

\section{Applications to First-Order Dynamic Equations on Time Scales}

Definition 59 (see [5]). Let $A(t)$ be $m \times m$ rd-continuous matrix-valued function on $\mathbb{T}$. The linear system

$$
x^{\Delta}(t)=A(t) x(t), \quad t \in \mathbb{T}
$$

is said to admit an exponential dichotomy on $\mathbb{T}$ if there exist positive constants $K, \alpha$, projection $P$, and the fundamental solution matrix $X(t)$ of (65) satisfying

$$
\begin{gathered}
\left|X(t) P X^{-1}(s)\right|_{\mathbb{T}} \leq K e_{\ominus \alpha}(t, s), \quad s, t \in \mathbb{T}, t \geq s, \\
\left|X(t)(I-P) X^{-1}(s)\right|_{\mathbb{T}} \leq K e_{\ominus \alpha}(s, t), \quad s, t \in \mathbb{T}, s \geq t,
\end{gathered}
$$

where $|\cdot|_{\mathbb{T}}$ is the matrix norm on $\mathbb{T}$. This means that if $A=$ $\left(a_{i j}\right)_{m \times m}$ then we can take $|A|_{\mathbb{T}}=\left(\sum_{i=1}^{m} \sum_{j=1}^{m}\left|a_{i j}\right|^{2}\right)^{1 / 2}$.

Consider the following system:

$$
x^{\Delta}(t)=A(t) x(t)+f(t), \quad t \in \mathbb{T},
$$

where $A$ is an $m \times m$ matrix-valued function which is regressive on $\mathbb{T}$ and $f: \mathbb{T} \rightarrow \mathbb{R}^{m}$ is rd-continuous.

Theorem 60 (see [11]). Let $\mathbb{T}$ be a symmetric time scale which is invariant under translation and let $A \in \mathscr{R}\left(\mathbb{T}, \mathbb{R}^{m \times m}\right)$ be almost automorphic and nonsingular on $\mathbb{T}$ and $\left(A^{-1}(t)\right)_{t \in \mathbb{T}}$ and $\left\{(I+\mu(t) A(t))^{-1}\right\}_{t \in \mathbb{T}}$ are bounded. Assume that linear system (65) admits an exponential dichotomy and $f \in C_{\mathrm{rd}}\left(\mathbb{T}, \mathbb{R}^{m}\right)$ is an almost automorphic function on time scales. Then system (67) has an almost automorphic solution as follows:

$$
\begin{aligned}
x(t)= & \int_{-\infty}^{t} X(t) P X^{-1}(\sigma(s)) f(s) \Delta s \\
& -\int_{t}^{\infty} X(t)(I-P) X^{-1}(\sigma(s)) f(s) \Delta s,
\end{aligned}
$$

where $X(t)$ is the fundamental solution matrix of (65).

Lemma 61 (see [3]). Let $c_{i}: \mathbb{T} \rightarrow(0,+\infty)$ be an almost automorphic function, $-c_{i} \in \mathscr{R}^{+}$, and $\min _{1 \leq i \leq m} \inf _{t \in \mathbb{T}}\left\{c_{i}(t)\right\}>$ 0 . Then the linear system

$$
x^{\Delta}(t)=\operatorname{diag}\left(-c_{1}(t),-c_{2}(t), \ldots,-c_{m}(t)\right) x(t)
$$

admits an exponential dichotomy on $\mathbb{T}$.

In view of Lemma 61 and Theorem 60 we have the following result.

Lemma 62. Let $\mathbb{T}$ be a symmetric time scale which is invariant under translation. Let $A(t)=\operatorname{diag}\left(-c_{1}(t),-c_{2}(t), \ldots,-c_{m}(t)\right)$ be such that the functions $c_{i}: \mathbb{T} \rightarrow(0,+\infty) i=1,2, \ldots, m$ are almost automorphic, $-c_{i} \in \mathscr{R}^{+}$, and $\min _{1 \leq i \leq m} \inf _{t \in \mathbb{T}}\left\{c_{i}(t)\right\}>0$, $i=1,2, \ldots, m$. Assume also that $f \in C_{\mathrm{rd}}\left(\mathbb{T}, \mathbb{R}^{m}\right)$ is an almost automorphic function on time scales. Then system (67) has an almost automorphic solution as follows:

$$
x_{i}(t)=\int_{-\infty}^{t} e_{-c_{i}}(t, \sigma(s)) f_{i}(s) \Delta s, \quad i=1,2, \ldots, m .
$$

Lemma 63. Let $\mathbb{T}$ be a symmetric time scale which is invariant under translation. Let $A(t)=\operatorname{diag}\left(-c_{1}(t),-c_{2}(t), \ldots,-c_{m}(t)\right)$ be such that the functions $c_{i}: \mathbb{T} \rightarrow(0,+\infty) i=1,2, \ldots, m$ are $C_{\mathrm{rd}}^{1}$-almost automorphic, $-c_{i} \in \mathscr{R}^{+}$, and $\min _{1 \leq i \leq m} \inf _{t \in \mathbb{T}}$ $\left\{c_{i}(t)\right\}>0, i=1,2, \ldots, m$. Assume also that $f$ is a $C_{\mathrm{rd}}^{1}-$ almost automorphic function on time scales. Then system (67) has a $C_{\mathrm{rd}}^{1}$-almost automorphic solution as follows:

$$
x_{i}(t)=\int_{-\infty}^{t} e_{-c_{i}}(t, \sigma(s)) f_{i}(s) \Delta s, \quad i=1,2, \ldots, m .
$$

Proof. By Lemma 62,

$$
x_{i}(t)=\int_{-\infty}^{t} e_{-c_{i}}(t, \sigma(s)) f_{i}(s) \Delta s, \quad i=1,2, \ldots, m,
$$

is a $C_{\mathrm{rd}}$-almost automorphic solution to system (67). Now, since

$$
\begin{aligned}
x_{i}^{\Delta}(t)= & -c_{i}(t) \int_{-\infty}^{t} e_{-c_{i}}(t, \sigma(s)) f_{i}(s) \Delta s \\
& +f_{i}(t), \quad i=1,2, \ldots, m,
\end{aligned}
$$


using on the one hand the fact that functions $t \mapsto \int_{-\infty}^{t} e_{-c_{i}}$ $(t, \sigma(s)) f_{i}(s) \Delta s, t \mapsto c_{i}(t)$, and $t \mapsto f_{i}$ are $C_{\text {rd }}$-almost automorphic and on the other hand the fact that Theorem 35 holds, we deduce that $x_{i}^{\Delta}(t), i=1,2, \ldots, m$, are also $C_{\mathrm{rd}^{-}}$ almost automorphic. The proof of Lemma 63 is then complete.

In the following we will consider $A A_{\mathbb{T}}^{(1)}(\mathbb{R})$ with the norm $\|\cdot\|_{1}$ obtained by taking $n=1$ in (37).

Set $E=\left\{\psi=\left(\psi_{1}, \psi_{2}, \ldots, \psi_{m}\right)^{T} \mid \psi_{i} \in A A_{\mathbb{T}}^{(1)}(\mathbb{R}), i=\right.$ $1,2, \ldots, m\}$. Then with the norm $\|\psi\|_{E}=\max _{1 \leq i \leq m}\|\psi\|_{1}, E$ is a Banach space.

Definition 64. Let $z^{*}(t)=\left(x_{1}^{*}(t), x_{2}^{*}(t), \ldots, x_{m}^{*}(t)\right)^{T}$ be a $C_{\mathrm{rd}}^{1}$-almost automorphic solution of (2) with initial value $\varphi^{*}(s)=\left(\varphi_{1}^{*}(t), \varphi_{2}^{*}(t), \ldots, \varphi_{m}^{*}(t)\right)^{T}$. Assume that there exists a positive constant $\lambda$ with $-\lambda \in \mathscr{R}^{+}$such that for $t_{0} \in$ $[-\theta, 0]_{\mathbb{T}}$, there exists $M>1$ such that for an arbitrary solution $z(t)=\left(x_{1}(t), x_{2}(t), \ldots, x_{m}(t)\right)^{T}$ of $(2)$ with initial value $\varphi(t)=$ $\left(\varphi_{1}(t), \varphi_{2}(t), \ldots, \varphi_{m}(t)\right)^{T}, z$ satisfies

$$
\begin{array}{r}
\left\|z(t)-z^{*}(t)\right\|_{1} \leq M\left\|\varphi-\varphi^{*}\right\|_{E} e_{-\lambda}\left(t, t_{0}\right), \\
t \in[-\theta,+\infty)_{\mathbb{T}}, \quad t \geq t_{0},
\end{array}
$$

where

$$
\begin{aligned}
& \mid\left\|z(t)-z^{*}(t)\right\|_{1} \\
& =\max _{1 \leq i \leq m}\left[\left|x_{i}(t)-x_{i}^{*}(t)\right|+\left|\left(x_{i}-x_{i}^{*}\right)^{\Delta}(t)\right|\right], \\
& \left\|\varphi-\varphi^{*}\right\|_{E} \\
& =\max _{1 \leq i \leq m} \sup _{t \in[-\theta, 0]_{\top}}\left(\left|\varphi_{i}(t)-\varphi_{i}^{*}(t)\right|+\left|\left(\varphi_{i}-\varphi_{i}^{*}\right)^{\Delta}(t)\right|\right) .
\end{aligned}
$$

Then, the solution $z^{*}$ is said to be exponentially stable.

In what follows, we will give sufficient condition for the existence of $C_{\mathrm{rd}}^{n}$-almost automorphic solutions of (2).

Let

$$
\begin{array}{r}
E=\left\{\phi(t)=\left(\phi_{1}(t), \phi_{2}(t), \ldots, \phi_{m}(t)\right)^{T} \mid \phi_{i} \in A A_{\mathbb{T}}^{(1)}(\mathbb{R}),\right. \\
i=1,2, \ldots, m\} .
\end{array}
$$

Then it is clear that $E$ endowed with the norm $\|\phi\|_{E}=$ $\max _{1 \leq i \leq m}\left\|\phi_{i}\right\|_{1}$, where $\|\cdot\|_{1}$ is obtained by taking $n=1$ in (37), is a Banach space.

We make the following assumption.

$\left(H_{1}\right)$ Assume $a_{i}, b_{i j}, I_{i} \in A A_{\mathbb{T}}^{(1)}\left(\mathbb{R}^{+}\right), t-\tau_{i j} \in A A_{\mathbb{T}}^{(1)}(\mathbb{T}),-a_{i} \in$ $\mathscr{R}^{+}, i, j=1,2, \ldots, m$, and $\min _{1 \leq i \leq m} \inf _{t \in \mathbb{T}}\left\{a_{i}(t)\right\}>0$.

$\left(H_{2}\right)$ There exists a positive constant $M_{j}, j=1,2, \ldots, m$, such that

$$
\left|f_{j}(x)\right| \leq M_{j}, \quad j=1,2 \ldots, m .
$$

$\left(H_{3}\right) f_{j} \in A A_{\mathbb{T}}^{(1)}(\mathbb{R}), j=1,2, \ldots, m$, and there exists a positive constant $H_{j}^{f}$ such that

$$
\begin{array}{r}
\left|f_{j}(u)-f_{j}(v)\right| \leq H_{j}^{f}|u-v|, \\
\forall u, v \in \mathbb{R}, \quad j=1,2 \ldots, m .
\end{array}
$$

For convenience, for a $C_{\mathrm{rd}}^{1}$-almost automorphic function $f: \mathbb{T} \rightarrow \mathbb{R}$, we set $\bar{f}=\sup _{t \in \mathbb{\mathbb { T }}}|f(t)|$ and by $\underline{f}=\inf _{t \in \mathbb{T}}|f(t)|$.

Theorem 65. Assume that $\left(H_{1}\right)$ and $\left(H_{3}\right)$ hold. Assume also that

$$
\max _{1 \leq i \leq m}\left[\frac{1}{\underline{a_{i}}} \sum_{j=1}^{m} \overline{b_{i j}} H_{j}^{f},\left(1+\frac{\overline{a_{i}}}{\underline{a_{i}}}\right) \sum_{j=1}^{m} \overline{b_{i j}} H_{j}^{f}\right]<1 .
$$

Then, (2) has a unique $C_{\mathrm{rd}}^{1}$-almost automorphic solution in $E_{0}=\left\{\phi(t) \in E \mid\|\phi\|_{E} \leq L\right\}$, where $L$ is a positive constant satisfying

$$
L=\max _{1 \leq i \leq m}\left(\sum_{j=1}^{m} \overline{b_{i j}} M_{j}+\overline{I_{i}}\right)\left(1+\frac{\overline{a_{i}}+1}{\underline{a_{i}}}\right) .
$$

Proof. For any $\varphi \in E$, we consider the following $C_{\mathrm{rd}^{-}}^{1}$-almost automorphic system:

$$
\begin{aligned}
x_{i}^{\Delta}= & -a_{i}(t) x_{i}(t)+\sum_{j=1}^{m} b_{i j}(t) f_{j}\left(t, \varphi_{j}\left(t-\tau_{i j}(t)\right)\right) \\
& +I_{i}(t), \quad t \in \mathbb{T}, i=1,2, \ldots, m .
\end{aligned}
$$

Since $\left(H_{1}\right)$ holds, it follows from Lemma 61 that the system

$$
x_{i}^{\Delta}(t)=-a_{i}(t) x_{i}(t), \quad i=1,2, \ldots, m,
$$

admits an exponential dichotomy on $\mathbb{T}$. Thus by Lemma 63 , system (2) has a $C_{\text {rd }}^{1}$-almost automorphic solution:

$$
\begin{aligned}
& x_{i}^{\varphi}(t)=\int_{-\infty}^{t} e_{-a_{i}}(t, \sigma(s)) \\
& \times\left(\sum_{j=1}^{m} b_{i j}(s) f_{j}\left(\varphi_{j}\left(s-\tau_{i j}(s)\right)\right)+I_{i}(s)\right) \Delta s, \\
& i=1,2, \ldots, m .
\end{aligned}
$$

Now, we prove that the following mapping is a contraction on $E_{0}$ :

$$
\begin{aligned}
\Gamma: E & \longrightarrow E \\
\varphi= & \left(\varphi_{1}, \varphi_{2}, \ldots, \varphi_{m}\right)^{T} \\
& \longmapsto(\Gamma \varphi)(t)=\left[(\Gamma \varphi)_{1}(t),(\Gamma \varphi)_{2}(t), \ldots,(\Gamma \varphi)_{m}(t)\right]^{T},
\end{aligned}
$$


where

$$
\begin{array}{r}
(\Gamma \varphi)_{i}(t)=\int_{-\infty}^{t} e_{-a_{i}}(t, \sigma(s)) \\
\times\left(\sum_{j=1}^{m} b_{i j}(s) f_{j}\left(\varphi_{j}\left(s-\tau_{i j}(s)\right)\right)+I_{i}(s)\right) \Delta s, \\
i=1,2, \ldots, m .
\end{array}
$$

$$
\begin{aligned}
& +\left|\sum_{j=1}^{m} b_{i j}(t) f_{j}\left(\varphi_{j}\left(t-\tau_{i j}(t)\right)\right)+I_{i}(t)\right| \\
\leq & \frac{\overline{a_{i}}}{\underline{a_{i}}}\left(\sum_{j=1}^{m} \overline{b_{i j}} M_{j}+\overline{I_{i}}\right)+\left(\sum_{j=1}^{m} \overline{b_{i j}} M_{j}+\overline{I_{i}}\right) \\
= & \left(\sum_{j=1}^{m} \overline{b_{i j}} M_{j}+\overline{I_{i}}\right)\left(1+\frac{\overline{a_{i}}}{\underline{a_{i}}}\right) .
\end{aligned}
$$

To this end we proceed in two steps.

Step 1. We prove that if $\varphi \in E_{0}$ then $(\Gamma \varphi) \in E_{0}$.

Let $\varphi \in E_{0}$; then using $\left(H_{2}\right)$ and the fact that $b_{i j}, I_{i} \in$ $A A_{\mathbb{T}}^{(1)}(\mathbb{R})$, we have

$$
\begin{aligned}
\left|(\Gamma \varphi)_{i}(t)\right|= & \mid \int_{-\infty}^{t} e_{-a_{i}}(t, \sigma(s)) \\
& \times\left(\sum_{j=1}^{m} b_{i j}(s)\right. \\
& \left.\times f_{j}\left(\varphi_{j}\left(s-\tau_{i j}(s)\right)\right)+I_{i}(s)\right) \Delta s \mid \\
\leq & \int_{-\infty}^{t} e_{-a_{i}}(t, \sigma(s)) \\
& \times\left(\sum_{j=1}^{m} b_{i j}(s)\right. \\
& \left.+\mid-\sum_{j=1}^{m} \overline{b_{i j}} M_{j}+\overline{I_{i}}\right) \int_{-\infty}^{t} e_{-a_{i}}(t, \sigma(s)) \Delta s \\
\leq & \frac{1}{a_{i}}\left(\sum_{j=1}^{m} \overline{b_{i j}} M_{j}+\overline{I_{i}}\right), \\
\left|(\Gamma \varphi)_{i}^{\Delta}(t)\right| \leq & \left.\left|f_{j}\left(\varphi_{j}\left(s-\tau_{i j}(s)\right)\right)\right|+\left|I_{i}(s)\right|\right) \Delta s \\
& \times\left(\sum_{j=1}^{m} b_{i j}(s)\right. \\
a_{-\infty}(t, \sigma(s)) &
\end{aligned}
$$

Consequently,

$$
\begin{aligned}
\|(\Gamma \varphi)\|_{E} & =\max _{1 \leq i \leq m} \sup _{t \in \mathbb{T}}\left[\left|(\Gamma \varphi)_{i}(t)\right|+\left|(\Gamma \varphi)_{i}^{\Delta}(t)\right|\right] \\
& \leq \max _{1 \leq i \leq m}\left(\sum_{j=1}^{m} \overline{i_{i j}} M_{j}+\overline{I_{i}}\right)\left(1+\frac{\overline{a_{i}}+1}{\underline{a_{i}}}\right)=L .
\end{aligned}
$$

Step 2. We prove that $\Gamma$ is a contraction on $E_{0}$.

Let $\varphi$ and $\phi$ be in $E_{0}$. Then using $\left(H_{3}\right)$ and the fact that $b_{i j} \in A A_{\mathbb{T}}^{(1)}(\mathbb{R})$, we obtain

$$
\begin{aligned}
& \left|(\Gamma \varphi)_{i}(t)-(\Gamma \phi)_{i}(t)\right| \\
& =\mid \int_{-\infty}^{t} e_{-a_{i}}(t, \sigma(s)) \\
& \quad \times\left[\sum _ { j = 1 } ^ { m } b _ { i j } ( s ) \left(f_{j}\left(\varphi_{j}\left(s-\tau_{i j}(s)\right)\right)\right.\right. \\
& \left.\left.\quad-f_{j}\left(\phi_{j}\left(s-\tau_{i j}(s)\right)\right)\right)\right] \Delta s \mid \\
& \leq \int_{-\infty}^{t} e_{-a_{i}}(t, \sigma(s)) \\
& \times\left[\sum_{j=1}^{m} b_{i j}(s) \mid f_{j}\left(\varphi_{j}\left(s-\tau_{i j}(s)\right)\right)\right. \\
& \quad-f_{j}\left(\phi_{j}\left(s-\tau_{i j}(s)\right)\right) \mid \Delta s \\
& \leq \int_{-\infty}^{t} e_{-a_{i}}(t, \sigma(s))\left[\sum_{j=1}^{m} b_{i j}(s) H_{j}^{f} \mid \varphi_{j}\left(s-\tau_{i j}(s)\right)\right.
\end{aligned}
$$




$$
\begin{aligned}
& \leq\left[\sum_{j=1}^{m} \overline{b_{i j}} H_{j}^{f}\|\varphi-\phi\|_{E_{0}}\right] \int_{-\infty}^{t} e_{-a_{i}}(t, \sigma(s)) \Delta s \\
& \leq \frac{1}{\underline{a_{i}}}\left[\sum_{j=1}^{m} \overline{b_{i j}} H_{j}^{f}\right]\|\varphi-\phi\|_{E_{0}}, \\
& \left|(\Gamma \varphi)_{i}^{\Delta}(t)-(\Gamma \phi)_{i}^{\Delta}(t)\right| \\
& \leq \mid-a_{i}(t) \int_{-\infty}^{t} e_{-a_{i}}(t, \sigma(s)) \\
& \times\left[\sum _ { j = 1 } ^ { m } b _ { i j } ( s ) \left(f_{j}\left(\varphi_{j}\left(s-\tau_{i j}(s)\right)\right)\right.\right. \\
& \left.\left.-f_{j}\left(\phi_{j}\left(s-\tau_{i j}(s)\right)\right)\right)\right] \Delta s \\
& +\mid \sum_{j=1}^{m} b_{i j}(t)\left(f_{j}\left(\varphi_{j}\left(s-\tau_{i j}(s)\right)\right)\right. \\
& \left.-f_{j}\left(\phi_{j}\left(s-\tau_{i j}(s)\right)\right)\right) \\
& \leq \underline{\underline{a_{i}}}\left[\sum_{j=1}^{m} \overline{b_{i j}} H_{j}^{f}\|\varphi-\phi\|_{E_{0}}\right]+\left[\sum_{j=1}^{m} \overline{b_{i j}} H_{j}^{f}\|\varphi-\phi\|_{E_{0}}\right] \\
& =\left[\sum_{j=1}^{m} \overline{b_{i j}}(s) H_{j}^{f}\right]\left[1+\frac{\overline{a_{i}}}{\underline{a_{i}}}\right]\|\varphi-\phi\|_{E_{0}} .
\end{aligned}
$$

Hence,

$$
\begin{aligned}
& \|(\Gamma \varphi)-(\Gamma \phi)\|_{E} \\
& =\max _{1 \leq i \leq m} \sup _{t \in \mathbb{T}}\left[\left|(\Gamma \varphi)_{i}(t)-(\Gamma \phi)_{i}(t)\right|\right. \\
& \left.\quad+\left|(\Gamma \varphi)_{i}^{\Delta}(t)-(\Gamma \phi)_{i}^{\Delta}(t)\right|\right] \\
& \leq \max _{1 \leq i \leq m}\left[\frac{1}{\underline{a_{i}}} \sum_{j=1}^{m} \overline{b_{i j}} H_{j}^{f},\left(1+\frac{\overline{a_{i}}}{\underline{a_{i}}}\right) \sum_{j=1}^{m} \overline{b_{i j}} H_{j}^{f}\right]\|\varphi-\phi\|_{E_{0}} .
\end{aligned}
$$

Because $\max _{1 \leq i \leq m}\left[\left(1 / \underline{a_{i}}\right) \sum_{j=1}^{m} \overline{b_{i j}} H_{j}^{f},\left(1+\left(\overline{a_{i}} / \underline{a_{i}}\right)\right) \sum_{j=1}^{m} \overline{b_{i j}}\right.$ $\left.H_{j}^{f}\right]<1, \Gamma$ is a contraction on $E_{0}$. We then deduce by the fixed point theorem of Banach that $\Gamma$ has a unique solution in $E_{0}$. Consequently system (2) has a unique $C_{\mathrm{rd}}^{1}$-almost automorphic solution in $E_{0}$ :

$$
\begin{aligned}
& x_{i}^{\varphi}(t)=\int_{-\infty}^{t} e_{-a_{i}}(t, \sigma(s)) \\
& \times\left(\sum_{j=1}^{m} b_{i j}(s) f_{j}\left(\varphi_{j}\left(s-\tau_{i j}(s)\right)\right)+I_{i}(s)\right) \Delta s, \\
& i=1,2, \ldots, m .
\end{aligned}
$$

Theorem 66. Assume that $\left(H_{1}\right)$ and $\left(H_{3}\right)$ hold. Assume also that

$$
\left(H_{4}\right): \theta_{i}=\sum_{j=1}^{m} \overline{b_{i j}} H_{j}^{f} \geq \frac{a_{i}-\underline{a_{i} \overline{a_{i}}}}{\underline{a_{i}}+\overline{a_{i}}}, \quad i=1,2, \ldots, m .
$$

Then, the $C_{\mathrm{rd}}^{1}$-almost automorphic solution of (2) is globally exponentially stable.

Proof. According to Theorem 65, system (2) has a $C_{\text {rd }}^{1}$-almost automorphic solution $x^{*}(t)=\left(x_{1}^{*}(t), x_{2}^{*}(t), \ldots, x_{m}^{*}(t)\right)^{T}$ with initial value

$$
\varphi^{*}(s)=\left(\varphi_{1}^{*}(t), \varphi_{2}^{*}(t), \ldots, \varphi_{m}^{*}(t)\right)^{T} .
$$

Assume that $z(t)=\left(x_{1}(t), x_{2}(t), \ldots, x_{m}(t)\right)^{T}$ is an arbitrary solution of (2) with initial value $\varphi(t)=\left(\varphi_{1}(t)\right.$, $\left.\varphi_{2}(t), \ldots, \varphi_{m}(t)\right)^{T}$. Let $y_{i}(t)=x_{i}(t)-x_{i}^{*}(t), i=1,2, \ldots, m$. Then it follows from (2) that

$$
\begin{array}{r}
y_{i}^{\Delta}(t)=-a_{i}(t) y_{i}(t)+\sum_{j=1}^{m} b_{i j}(t)\left[f_{j}\left(x_{j}\left(t-\tau_{i j}(t)\right)\right)\right. \\
\left.-f_{j}\left(x_{j}^{*}\left(t-\tau_{i j}(t)\right)\right)\right], \\
t \in \mathbb{T}, \quad i=1,2, \ldots, m, \\
y_{i}(s)=\varphi(s)-\varphi^{*}(s), \quad s \in[-\theta, 0]_{\mathbb{T}}, \quad i=1,2, \ldots, m .
\end{array}
$$

Multiplying both sides of the first equation in (93) by $e_{-a_{i}}$ $(t, \sigma(s))$ and integrating on $\left[t_{0}, t\right]_{\mathbb{T}}$, where $t_{0} \in[-\theta, 0]_{\mathbb{T}}$, we obtain, for $i=1,2, \ldots, m$,

$$
\begin{aligned}
& \int_{t_{0}}^{t} y_{i}^{\Delta}(s) e_{-a_{i}}(t, \sigma(s)) \Delta s \\
& =\int_{t_{0}}^{t}-a_{i}(s) y_{i}(s) e_{-a_{i}}(t, \sigma(s)) \Delta s \\
& +\sum_{j=1}^{m} \int_{t_{0}}^{t} b_{i j}(s)\left[f_{j}\left(x_{j}\left(s-\tau_{i j}(s)\right)\right)\right. \\
& \left.\quad-f_{j}\left(x_{j}^{*}\left(s-\tau_{i j}(s)\right)\right)\right] e_{-a_{i}}(t, \sigma(s)) \Delta s .
\end{aligned}
$$


This means that, for $i=1,2, \ldots, m$,

$$
\begin{aligned}
y_{i}(t)=y_{i}\left(t_{0}\right) e_{-a_{i}}\left(t, t_{0}\right) & \\
+\sum_{j=1}^{m} \int_{t_{0}}^{t} b_{i j}(s) & {\left[f_{j}\left(x_{j}\left(s-\tau_{i j}(s)\right)\right)\right.} \\
& \left.-f_{j}\left(x_{j}^{*}\left(s-\tau_{i j}(s)\right)\right)\right] e_{-a_{i}}(t, \sigma(s)) \Delta s .
\end{aligned}
$$

Then choose $\lambda<\min _{1 \leq i \leq m} \underline{a_{i}}$ and

$$
M>\max _{1 \leq i \leq m}\left\{\frac{\underline{a_{i} \overline{a_{i}}}}{\underline{a_{i}}-\left(\underline{a_{i}}+\overline{a_{i}}\right) \theta_{i}}, \frac{\underline{a_{i}}}{\underline{a_{i}}-\theta_{i}}\right\} .
$$

One proves by contradiction as in [8] that

$$
|\|y(t)\||_{1} \leq M e_{\ominus \lambda}\left(t, t_{0}\right)\left\|\varphi-\varphi^{*}\right\|_{E}, \quad \forall t \in\left(t_{0},+\infty\right)_{\mathbb{T}} .
$$

This means that the $C_{\mathrm{rd}}^{1}$-almost automorphic solution $x^{*}$ of (2) is globally exponentially stable.

\section{Conflict of Interests}

The authors declare that there is no conflict of interests regarding the publication of this paper.

\section{Acknowledgments}

The authors would like to thank the referee for his/her careful reading and valuable suggestions. Aril Milce received a Ph.D. scholarship from the French Embassy in Haiti. He is also supported by "Ecole Normale Supérieure," an entity of the "Université d'Etat d'Haïti."

\section{References}

[1] S. Hilger, Ein Makettenkalkül mit Anwendung auf Zentrumsmannigfaltigkeiten [Ph.D. thesis], Universität Würzburg, 1988.

[2] C. Lizama, J. G. Mesquita, and R. Ponce, "A connection between almost periodic functions defined on time scales and $\mathbb{R}$," Applicable Analysis, vol. 93, no. 12, pp. 2547-2558, 2014.

[3] Y. Li and C. Wang, "Almost periodic functions on time scales and applications," Discrete Dynamics in Nature and Society, vol. 2011, Article ID 727068, 20 pages, 2011.

[4] Y. Li and L. Yang, "Almost periodic solutions for neutral-type BAM neural networks with delays on time scales," Journal of Applied Mathematics, vol. 2013, Article ID 942309, 13 pages, 2013.

[5] Y. Li and C. Wang, "Uniformly almost periodic functions and almost periodic solutions to dynamic equations on time scales," Abstract and Applied Analysis, vol. 2011, Article ID 341520, 22 pages, 2011.

[6] J. Zhang, M. Fan, and H. Zhu, "Existence and roughness of exponential dichotomies of linear dynamic equations on time scales," Computers and Mathematics with Applications, vol. 59, no. 8, pp. 2658-2675, 2010.
[7] V. Lakshmikantham and A. S. Vatsala, "Hybrid systems on time scales," Journal of Computational and Applied Mathematics, vol. 141, no. 1-2, pp. 227-235, 2002.

[8] L. Yang, Y. Li, and W. Wu, " $C^{n}$-almost periodic functions and an application to a Lasota-Wazewka model on time scales," Journal of Applied Mathematics, vol. 2014, Article ID 321328, 10 pages, 2014.

[9] M. Bohner and A. Peterson, Dynamic Equations on Time Scales. An Introduction with Applications, Birkhäuser, Boston, Mass, USA, 2001.

[10] M. Bohner and A. Peterson, Advances in Dynamic Equations on Time Scales, Birkhäuser, Boston, Mass, USA, 2003.

[11] C. Lizama and J. G. Mesquita, "Almost automorphic solutions of dynamic equations on time scales," Journal of Functional Analysis, vol. 265, no. 10, pp. 2267-2311, 2013.

[12] G. M. N'Guérékata, Almost Automorphic and Almost Periodicity Functions in Abstract Spaces, Kluwer Academic, New York, NY, USA, 2001 


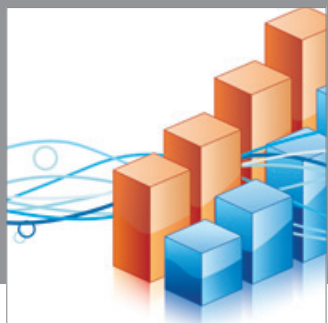

Advances in

Operations Research

mansans

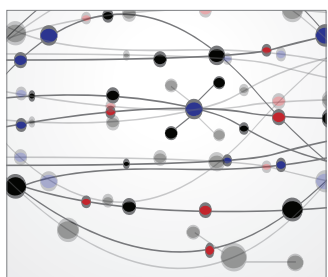

The Scientific World Journal
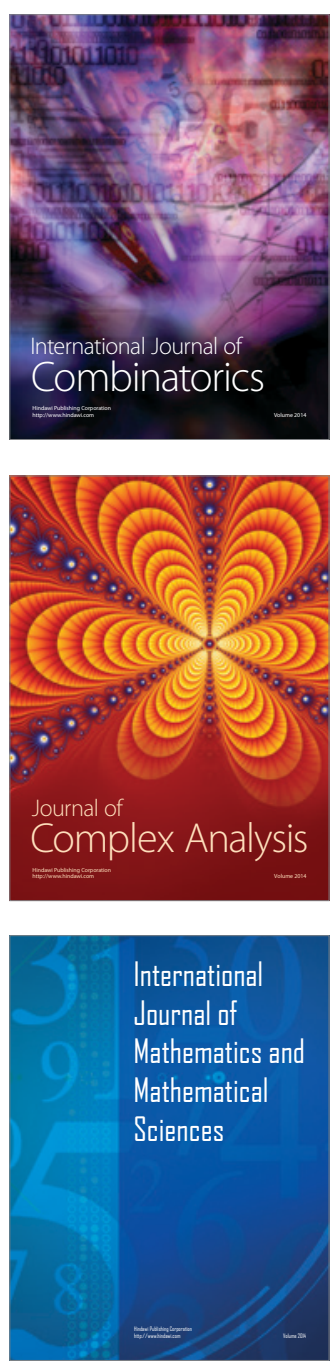
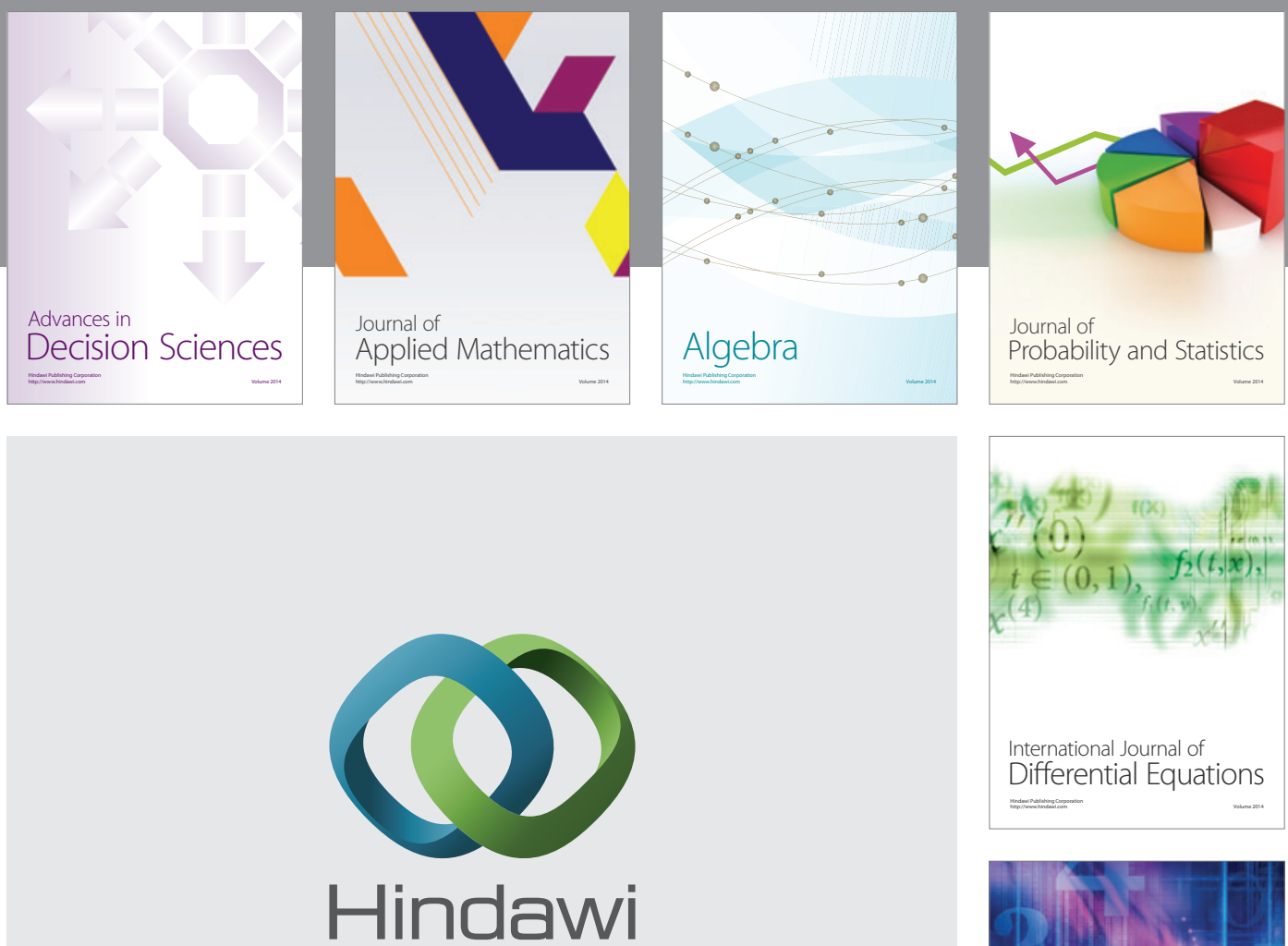

Submit your manuscripts at http://www.hindawi.com
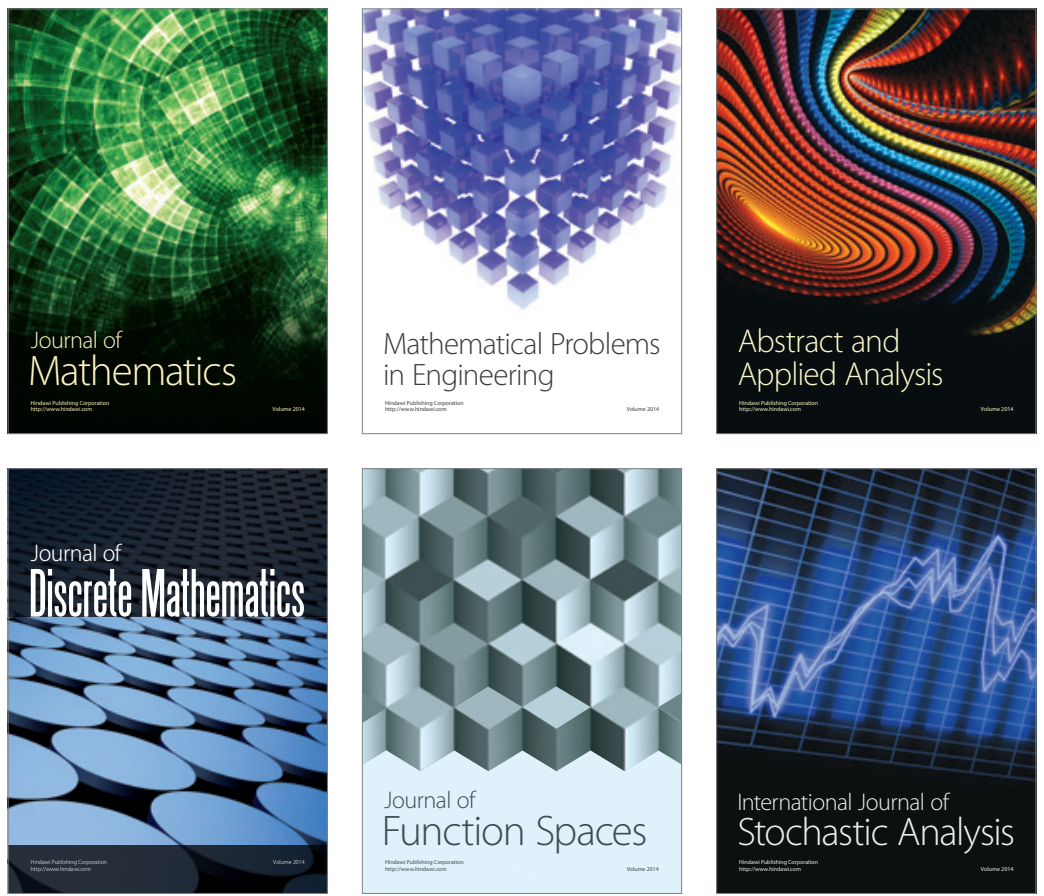

Journal of

Function Spaces

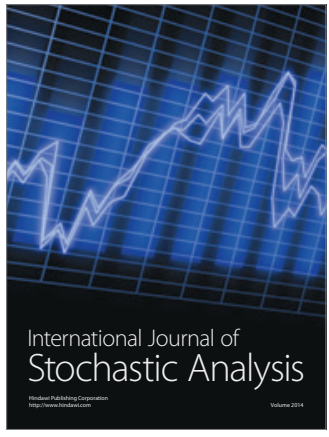

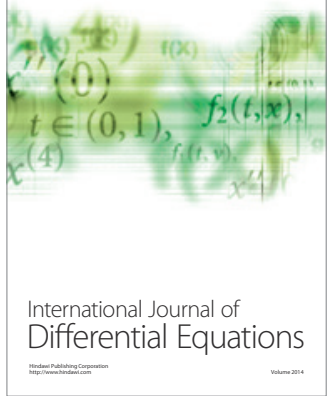
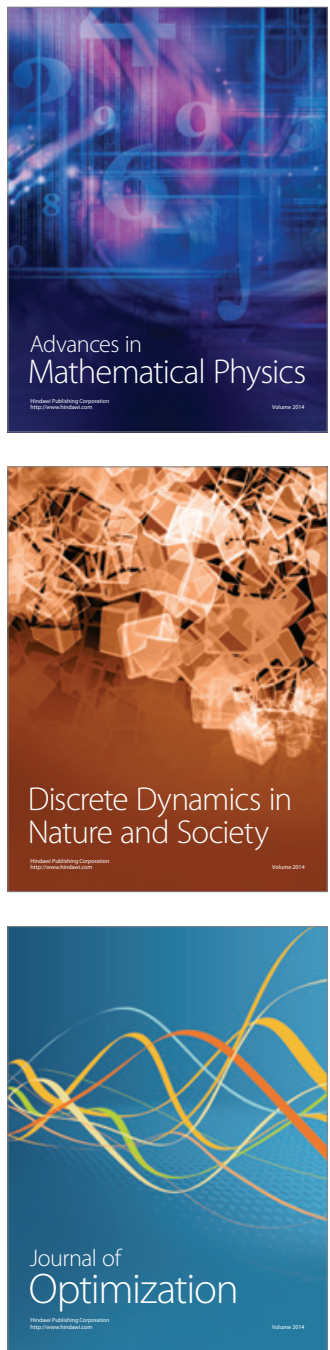